\title{
An oily fish diet increases insulin sensitivity compared to a red meat diet in young iron-deficient women
}

\author{
Santiago Navas-Carretero ${ }^{1}$, Ana M. Pérez-Granados ${ }^{1}$, Stefanie Schoppen ${ }^{1,2}$ and M. Pilar Vaquero ${ }^{1 *}$ \\ ${ }^{1}$ Department of Metabolism and Nutrition, Instituto del Frío, Institute of Food Science and Technology and Nutrition (ICTAN), \\ Spanish National Research Council (CSIC), José Antonio Novais, 10, 28040 Madrid, Spain \\ ${ }^{2}$ Lipid Research Unit, Fundación Jiménez Díaz, Avd. Reyes Católicos 2, 28040 Madrid, Spain
}

(Received 10 October 2008 - Revised 4 December 2008 - Accepted 4 December 2008 - First published online 12 February 2009)

Beneficial effects of $n-3$ fatty acids on a variety of physiological functions have been reported, but information related to the effects of oily fish consumed within a varied diet on glucose metabolism and diabetes risk is scarce. The objective of the study was to compare the effects of a diet rich in oily fish to those of a diet rich in red meat on lipid profile, oxidative status, glucose metabolism and insulin sensitivity in young, iron-deficient women. The study was designed attending the CONSORT statement guidelines. It was a randomised crossover dietary intervention study with two 8-week periods. Two diets were designed differing only in their oily fish or red meat content (four portions per week). Twenty-five young iron-deficient women with normal lipid, glucose and insulin levels participated in the assay. Lipid profile (total, LDL- and HDL-cholesterol, TAG), fasting glucose, fasting insulin, and oxidation (lipoperoxides) and inflammation (soluble intercellular adhesion molecule-1 and soluble vascular cell adhesion molecule-1) biomarkers were analysed. Insulin sensitivity was assessed by the Homeostasis Model Assessment (HOMA) and Quantitative Insulin Sensitivity Check Index (QUICKI). Insulin levels significantly decreased and insulin sensitivity significantly increased with the oily fish diet. HDL-cholesterol significantly increased with the oily fish diet. Other parameters did not significantly differ between diets. An increase in oily fish consumption increases insulin sensitivity in young iron-deficient women. This outcome should be considered when giving dietary advice to this population.

n-3 Fatty acids: Oily fish diet: Red meat: Insulin sensitivity

Consumption of oily fish is known to decrease the risk of CVD, primarily due to its $n-3$ fatty acid content ${ }^{(1)}$. The inverse association between fish intake and the risk of $\mathrm{CHD}$ has been reported in various cohort studies ${ }^{(2,3)}$. The mechanism by which $n-3$ fatty acids protect from CVD is related to the incorporation of EPA (20:5) and DHA (22:6) into membrane phospholipids. This inclusion produces changes in membrane properties ${ }^{(4,5)}$ with various beneficial effects: reduced activity of inflammatory cells and concentration of certain inflammation mediators ${ }^{(5)}$, reduced arterial plaque fragility ${ }^{(6)}$, regulation of blood pressure $^{(7)}$ and improved endothelial function in hypercholesterolaemic subjects ${ }^{(8)}$. A daily intake of 3-4 g EPA + DHA has also been associated with a reduction of serum $\mathrm{TAG}^{(9)}$, although other authors have observed that a lower $n-3$ fatty acid intake $(1.5-3.5 \mathrm{~g} / \mathrm{d})$ also reduces serum TAG in hyperlipaemic patients ${ }^{(10)}$. This TAG-lowering effect appears to be related to increased hepatic $\beta$-oxidation and decreased lipogenesis ${ }^{(11)}$.

Insulin resistance is considered an important risk factor for type 2 diabetes mellitus as well as an independent factor for CVD and the metabolic syndrome, even when glucose values are within the normal range ${ }^{(12)}$.

Various articles have described the inverse association of a high consumption of PUFA, from vegetables and seeds, with the risk of developing type 2 diabetes mellitus or the impairment of glucose and insulin metabolism, compared to that of saturated fat from animal products ${ }^{(13,14)}$.

The effects of high consumption of $n-3$ PUFA on glucose metabolism have been widely studied ${ }^{(15-17)}$, although the results with regard to insulin resistance are not conclusive. Most of the studies carried out on this issue have focused on supplementation with different doses of fish oils in healthy subjects or type 2 diabetes patients. Supplements of fish oils as high as $10 \mathrm{~g} / \mathrm{d}$ have reported negative effects on blood glucose levels and insulin function in diabetic patients ${ }^{(15)}$.

It has been observed that insulin sensitivity cannot be restored in the liver of type 2 diabetes patients, and that once type 2 diabetes mellitus is present, the potential benefits of $n$-3 PUFA intake do not seem to be effective ${ }^{(16,17)}$.

In addition, recent studies carried out in the USA and Europe have reported increased insulin sensitivity, as well as decreased fasting glucose levels, in healthy subjects consuming fish oil ${ }^{(18)}$. These studies have also associated the consumption of cod liver oil during pregnancy with protection from type 1 diabetes mellitus in the offspring ${ }^{(19)}$.

Although many intervention studies have focused on the effects of $n-3$ PUFA consumption as fish oil or supplements

Abbreviations: HDL-c, HDL-cholesterol; HOMA, Homeostasis Model Assessment; QUICKI, Quantitative Insulin Sensitivity Check Index; LDL-c,

LDL-cholesterol; sICAM-1, soluble intercellular adhesion molecule-1; sVCAM-1, vascular cell adhesion molecule-1; Total-c, total cholesterol.

* Corresponding author: Dr Ma Pilar Vaquero, fax +34915493627, email mpvaquero@if.csic.es; snavas@if.csic.es 
on glucose and insulin metabolism, data related to fish consumption and its role in the regulation of insulin resistance are scarce ${ }^{(10)}$.

Dietary advice for most of the population has recently been focused on reducing the intake of red meat and increasing fish consumption as a protective measure against developing CVD. The exception has been the advice to women at risk of iron deficiency, who have been encouraged to follow a diet rich in red meat.

On the other hand, it is known that iron overload leads to a number of metabolic disorders and the development of CVD, and reduction of iron stores improves glucose tolerance in type 2 diabetic patients ${ }^{(20)}$. A negative association has been observed between iron stores and insulin sensitivity in healthy subjects consuming a red meat-based diet compared to a lactoovo vegetarian diet ${ }^{(21)}$.

Taking into account these premises, the present study aimed at comparing the effects of two diets, one rich in oily fish and the other in red meat, but similar in all other components, on lipid profile, oxidative status, glucose and insulin levels, and insulin sensitivity, in young, iron-deficient women.

\section{Subjects and methods}

The present study was designed and carried out following the CONSORT statement guidelines ${ }^{(22)}$.

\section{Subjects}

Thirty women between the ages of 18 and 30, who had originally been recruited to form part of a wider study on the prevention of iron deficiency through diet, volunteered to participate in this nutritional intervention study.

After being informed of the study conditions and having signed an informed consent, the women underwent blood tests to determine their biochemical parameters. They also completed questionnaires relating to lifestyle, chronic diseases and medical treatments.

Inclusion criteria required that the women be menstruating, with low iron stores, and lipid, glucose and insulin levels within the normal ranges. Exclusion criteria were smoking, anaemia, to suffer from any chronic gastric disease, be allergic to fish or be vegetarian.

Of the thirty women who were initially interested in participating in the trial five did not complete the study, for which reason the final number of participants was twentyfive. All presented low iron stores (mean ferritin levels $<20 \mathrm{ng} / \mathrm{ml}$ ).

Study protocols were approved by the Ethics Committee of the Spanish National Research Council and the Clinical Research Ethics Committee of the Hospital Clinica Puerta de Hierro, both in Madrid, Spain.

\section{Study design}

The study consisted of a randomized crossover intervention involving two 8-week periods. The trial was held between the months of September and December. The twenty-five participants were randomly distributed into two groups of twelve and thirteen subjects, respectively.
Two diets, differing only in their red meat and oily fish content, were tested. Dietary instructions were given only in relation to meat, poultry, fish and egg intake. Volunteers were asked to maintain their usual dietary habits with regard to the rest of the food groups.

The 'red meat diet' contained five portions of red meat, one portion of lean fish, two portions of poultry and two eggs per week.

The 'oily fish diet' contained two portions of salmon, two cans of water-packed tuna ( $56 \mathrm{~g}$ each), one can of sardines in olive oil, one portion of lean fish, one portion of red meat, two portions of poultry and two eggs per week.

\section{Dietary control and compliance}

Each subject's dietary intake was regularly evaluated to control any possible changes in dietary patterns, including those related to the consumption of red meat or oily fish to detect possible dietary changes that could interfere with the study. Volunteers were asked to fill out a $24 \mathrm{~h}$ dietary recall with the menus they consumed every day, focusing on the ingredients. Once per month they completed a $72 \mathrm{~h}$ detailed intake report, specifying the types of food consumed and serving weights. Compliance was assured by the daily menu forms, as well as weekly interviews of volunteers by one member of the research team at the time of collection of the daily records. Dietary food and nutrient intakes, and energy provided by macronutrients were calculated by an informatic application using the Spanish Food Composition Database (DIAL, Alce Ingenieria, Spain). Compliance of the intervention was also assessed by personal telephone interviews throughout the whole intervention.

\section{Anthropometric measures and blood pressure}

Weight and height of each volunteer were measured, and BMI calculated, at 09.00 hours on day 0 (baseline) and on weeks 4 and 8 of each intervention period, after a $12 \mathrm{~h}$ fasting period. At the same time, blood pressure was measured by an automated manometer (Omron M3-intellisense; OMRON Healthcare Europe, Spain).

\section{Blood sampling and biochemical assays}

Volunteers reported to the laboratory facilities at baseline (day 0 ) and week 8 of each period. Blood samples were collected by venepuncture between 08.00 and 08.30 hours, after a $12 \mathrm{~h}$ fasting period. Serum and plasma were obtained after centrifugation at $1000 \mathrm{~g}$ for $30 \mathrm{~min}$ and stored at $-80^{\circ} \mathrm{C}$.

Serum total cholesterol (Total-c), HDL-cholesterol (HDL-c) and TAG concentrations were determined by automated enzymatic methods (CHOD-PAP and GPO-PAP, Boehringer Mannheim, Germany; RA-XT autoanalyser, Technicon, USA). Serum LDL-cholesterol (LDL-c) concentration was calculated using the Friedewald formula ${ }^{(23)}$. Total-c/HDL-c and LDL-c/ LDL-c ratios were calculated.

Total lipoperoxides (malondialdehyde and hydroxyalkenes) in blood were determined by quantitative colorimetry using a commercially available kit (Bioxytech LPO-586; Oxis Research, USA). Soluble intercellular adhesion molecule-1 (sICAM-1) and soluble vascular cell adhesion molecule-1 
(sVCAM-1) serum concentrations were measured by the ELISA technique, using a commercially available kit (Quantikine Human sVCAM-1 and sICAM-1, Parameter; R\&D Systems, USA).

Fasting serum glucose concentrations were analysed by an automatic analyser (RA 2000; Technicon, USA). Serum insulin levels were determined by means of an immunometric assay in an autoanalyser (Immulite ${ }^{\circledR} 2000$ Insulin; Diagnostic Products Corporation-DPC, UK).

Insulin sensitivity was estimated using the Homeostasis Model Assessment (HOMA) and the Quantitative Insulin Sensitivity Check Index (QUICKI), which were calculated using the following formulas:

$$
\begin{aligned}
\text { HOMA }= & (\text { fasting insulin }(\mu \mathrm{U} / \mathrm{ml}) \\
& \times \text { fasting glucose }(\mathrm{mmol} / \mathrm{l})) / 22 \cdot 5 \\
\text { QUICKI }= & 1 /(\log (\text { fasting insulin }(\mu \mathrm{U} / \mathrm{ml})) \\
& +\log (\text { fasting glucose }(\mathrm{mg} / \mathrm{dl})))
\end{aligned}
$$

To compare the effect of both dietary treatments, changes between baseline values and the values at the end of each period ( 8 weeks) were calculated as follows:

$$
\begin{aligned}
\text { Change }(\%)= & ((\text { Value at } 8 \text { weeks } \\
& - \text { Value at baseline }) / \text { Value at baseline }) \times 100
\end{aligned}
$$

\section{Statistics}

The SPSS 15.0 statistical package for Windows (SPSS Inc., Chicago, IL, USA) was used to study the data. Sample stratified randomization was made with Excel (Microsoft Office 2003; Microsoft Inc., Redmond, WA, USA).

Data are presented as means and standard deviations. Percentage change data were arcsin transformed when necessary. Data were analysed with a univariant ANOVA to study the influence of dietary treatment (fixed effects), the group to which volunteers were assigned and the order in which they started the intervention study (random effects). Once significant differences due to group and order were discarded, the data were analysed by a mixed model and multivariate ANOVA (fixed factor: type of diet). Values of $P \leq 0.05$ were considered to be statistically significant.

\section{Results}

Baseline parameters of the groups starting with either the red meat or the oily fish diet are shown in Table 1. No significant differences between them were observed at the beginning of the nutritional intervention.

Compliance was confirmed by the analysis of dietary reports. The two diets varied significantly only with regard to meat and fish consumption (Table 2). Intake of the food groups including cereals, legumes, vegetables, fruits, dairy products, eggs, sugars and oils was similar in both diets. In relation to nutrient intake (Table 3 ), no significant differences between diets were observed in total energy consumed or energy from macronutrients. The only significant differences between diets were those derived from oily fish consumption. With the oily fish diet, intake of $n-3$ PUFA, mainly in the form of EPA (20:5) and DHA (22:6), was significantly higher than with the red meat diet. Consequently, the PUFA/SFA ratio was also significantly higher while the $n-6 / n-3$ PUFA ratio was $50 \%$ lower than with the red meat diet. In contrast, intake of oleic acid, which contributes to more than $90 \%$ of MUFA, was significantly higher during the red meat diet. SFA intake did not significantly differ between the diets.

Body weight, BMI $\left(22 \cdot 1(\mathrm{SD} 2 \cdot 2) \mathrm{kg} / \mathrm{m}^{2}\right)$ and blood pressure remained constant during the entire intervention study.

Total-c and LDL-c levels decreased equally with both diets (Table 4), while HDL-c levels increased with both diets, the change being significantly higher with the oily fish diet than with the red meat one. Total-c/HDL-c and LDL-c/HDL-c

\begin{tabular}{|c|c|c|c|c|c|}
\hline & \multicolumn{2}{|c|}{$\begin{array}{l}\text { Group starting with } \\
\text { red meat diet }\end{array}$} & \multicolumn{2}{|c|}{$\begin{array}{l}\text { Group starting with } \\
\text { oily fish diet }\end{array}$} & \multirow[b]{2}{*}{ ANOVA } \\
\hline & Mean & SD & Mean & SD & \\
\hline Total cholesterol (mmol/l) & 4.51 & 0.79 & 4.46 & 0.82 & NS \\
\hline HDL-cholesterol (mmol/l) & 1.72 & 0.31 & 1.62 & 0.19 & NS \\
\hline LDL-cholesterol (mmol/l) & $2 \cdot 44$ & 0.75 & $2 \cdot 57$ & 0.82 & NS \\
\hline TAG $(\mathrm{mmol} / \mathrm{l})$ & 0.76 & 0.07 & 0.59 & 0.16 & NS \\
\hline Glucose (mmol/l) & 4.69 & 0.22 & 4.75 & 0.24 & NS \\
\hline Insulin (mU/l) & $5 \cdot 56$ & $1 \cdot 20$ & 6.59 & 2.92 & NS \\
\hline HOMA & $1 \cdot 21$ & 0.37 & 1.37 & 0.54 & NS \\
\hline QUICKI & 0.72 & 0.14 & 0.69 & $0 \cdot 19$ & NS \\
\hline Lipoperoxides $(\mu \mathrm{mol} / \mathrm{l})$ & $100 \cdot 3$ & $26 \cdot 9$ & $129 \cdot 2$ & 33.8 & NS \\
\hline sVCAM-1(ng/ml) & $599 \cdot 8$ & $201 \cdot 4$ & $537 \cdot 1$ & $72 \cdot 4$ & NS \\
\hline sICAM-1 (ng/ml) & $215 \cdot 5$ & $32 \cdot 7$ & $200 \cdot 0$ & $26 \cdot 9$ & NS \\
\hline
\end{tabular}
ratios tended to decrease to the same extent with both diets. A non-significant tendency for TAG levels to increase with

Table 1. Baseline biochemical parameters of volunteers in the group starting with the red meat diet and the group starting with the oily fish diet

(Mean values and standard deviations)

HOMA, Homeostasis Model Assessment; QUICKI, Quantitative Insulin Sensitivity Check Index; sICAM-1, soluble intercellular adhesion molecule-1; sVCAM-1, vascular cell adhesion molecule-1. 
Table 2. Food group intake of the volunteers during the oily fish or red meat diets (four portions per week)* (Mean values and standard deviations)

\begin{tabular}{|c|c|c|c|c|c|c|c|c|c|c|}
\hline \multirow[b]{3}{*}{ Food group (g/d) } & \multicolumn{4}{|c|}{ Oily fish diet } & \multicolumn{4}{|c|}{ Red meat diet } & & \\
\hline & \multicolumn{2}{|c|}{ 1st month } & \multicolumn{2}{|c|}{ 2nd month } & \multicolumn{2}{|c|}{ 1st month } & \multicolumn{2}{|c|}{ 2nd month } & \multicolumn{2}{|c|}{ ANOVA } \\
\hline & Mean & SD & Mean & SD & Mean & SD & Mean & SD & Diet & Time \\
\hline Cereals & 127 & 39 & 155 & 68 & 158 & 56 & 162 & 48 & NS & NS \\
\hline Legumes & 11 & 18 & 8 & 16 & 17 & 18 & 13 & 17 & NS & NS \\
\hline Vegetables & 215 & 103 & 175 & 97 & 196 & 103 & 214 & 129 & NS & NS \\
\hline Fruits & 127 & 78 & 122 & 89 & 127 & 78 & 110 & 100 & NS & NS \\
\hline Dairy products & 330 & 154 & 355 & 158 & 338 & 120 & 311 & 126 & NS & NS \\
\hline Meat products & 69 & 44 & 65 & 47 & 156 & 54 & 159 & 52 & $P<0.001$ & NS \\
\hline Fish products & 103 & 44 & 107 & 63 & 38 & 37 & 31 & 39 & $P<0.001$ & NS \\
\hline Eggs & 26 & 16 & 31 & 22 & 27 & 17 & 24 & 16 & NS & NS \\
\hline Sugars, candies and pastry & 24 & 29 & 32 & 27 & 20 & 14 & 15 & 11 & NS & NS \\
\hline Oils and fats & 44 & 16 & 42 & 21 & 46 & 13 & 52 & 16 & NS & NS \\
\hline
\end{tabular}

*For details of subjects and procedures, see the Subjects and methods section and Table 1.

the red meat diet and to decrease with the oily fish diet was observed.

Oxidation and inflammation parameters (lipoperoxides, sVCAM-1 and sICAM-1) displayed no significant differences between diets (Table 4), although lipoperoxide concentration displayed a marked tendency to increase with red meat diet.

Glucose levels displayed a slight tendency to decrease during the intervention, although the influence of diet was not significant (Fig. 1). Significant differences were seen between insulin levels, which were nearly $20 \%$ lower with the oily fish diet than with the red meat diet. As a result, HOMA and QUICKI indexes also improved significantly with the oily fish compared to the red meat diet.

\section{Discussion}

The results of the present study show that a high consumption of oily fish, rich in $n$-3 PUFA, increases insulin sensitivity compared to a diet rich in red meat in young iron-deficient women. Furthermore, the present study demonstrates that it is feasible to carry out a dietary intervention increasing oily fish intake within the framework of a varied diet and normal portion sizes.

The present study is part of a wider investigation about the effects of oily fish consumption on iron deficiency after our group previously observed that adding salmon to a phytaterich meal increases iron absorption ${ }^{(24)}$. In the present study,

Table 3. Macronutrient and micronutrient intake of the volunteers during the oily fish or red meat diets (four portions per week)*

(Mean values and standard deviations)

\begin{tabular}{|c|c|c|c|c|c|c|c|c|c|c|}
\hline & \multicolumn{4}{|c|}{ Red meat diet } & \multicolumn{4}{|c|}{ Oily fish diet } & & \\
\hline & \multicolumn{2}{|c|}{ 1st month } & \multicolumn{2}{|c|}{ 2nd month } & \multicolumn{2}{|c|}{ 1st month } & \multicolumn{2}{|c|}{ 2nd month } & \multicolumn{2}{|c|}{ ANOVA } \\
\hline & Mean & SD & Mean & SD & Mean & SD & Mean & SD & Diet & Time \\
\hline \multicolumn{11}{|l|}{ Energy } \\
\hline $\mathrm{kJ} / \mathrm{d}$ & 8541 & 1465 & 8738 & 1465 & 8093 & 1294 & 8809 & 1645 & NS & NS \\
\hline $\mathrm{kcal} / \mathrm{d}$ & 2040 & 350 & 2087 & 350 & 1933 & 309 & 2104 & 393 & NS & NS \\
\hline Carbohydrates (g/d) & 182 & 43 & 175 & 33 & 166 & 40 & 191 & 61 & NS & NS \\
\hline Protein $(g / d)$ & 83 & 17 & 80 & 15 & 76 & 13 & 81 & 17 & NS & NS \\
\hline Lipids (g/d) & 103 & 16 & 113 & 26 & 102 & 17 & 110 & 25 & NS & NS \\
\hline Fibre $(\mathrm{g} / \mathrm{d})$ & 17 & 4 & 15 & 5 & 15 & 3 & 15 & 5 & NS & NS \\
\hline $\operatorname{SFA}(g / d)$ & 32.5 & $7 \cdot 1$ & 33.6 & $10 \cdot 4$ & $30 \cdot 0$ & $8 \cdot 9$ & $32 \cdot 6$ & $12 \cdot 1$ & NS & NS \\
\hline MUFA (g/d) & $52 \cdot 7$ & $11 \cdot 5$ & 54.5 & $10 \cdot 9$ & $49 \cdot 8$ & $9 \cdot 6$ & $48 \cdot 3$ & $10 \cdot 2$ & NS & NS \\
\hline $18: 1(\mathrm{~g} / \mathrm{d})$ & $48 \cdot 4$ & $7 \cdot 9$ & $53 \cdot 4$ & $12 \cdot 6$ & $45 \cdot 8$ & $8 \cdot 0$ & $45 \cdot 9$ & 9.9 & $P=0.044$ & NS \\
\hline PUFA (g/d) & 11.9 & 3.4 & $12 \cdot 6$ & 3.5 & $13 \cdot 9$ & $5 \cdot 4$ & $15 \cdot 1$ & $4 \cdot 2$ & $P=0.013$ & NS \\
\hline n-6 PUFA (g/d) & $10 \cdot 8$ & $3 \cdot 3$ & $11 \cdot 7$ & $3 \cdot 8$ & $10 \cdot 9$ & $4 \cdot 4$ & $12 \cdot 6$ & $4 \cdot 4$ & NS & NS \\
\hline $18: 2(\mathrm{~g} / \mathrm{d})$ & $10 \cdot 7$ & $3 \cdot 2$ & $11 \cdot 6$ & $3 \cdot 8$ & $10 \cdot 6$ & 4.4 & $12 \cdot 3$ & $4 \cdot 4$ & NS & NS \\
\hline $20: 4(g / d)$ & 0.11 & 0.11 & 0.13 & 0.06 & 0.16 & 0.06 & 0.19 & 0.07 & $P=0.015$ & NS \\
\hline n-3 PUFA (g/d) & $1 \cdot 2$ & 0.4 & 1.4 & 0.5 & $2 \cdot 6$ & 0.8 & $3 \cdot 0$ & $1 \cdot 2$ & $P<0.001$ & NS \\
\hline $18: 3(g / d)$ & 1.0 & 0.3 & $1 \cdot 1$ & 0.4 & $1 \cdot 2$ & 0.4 & 1.4 & 0.5 & NS & NS \\
\hline $20: 5(g / d)$ & 0.05 & 0.09 & 0.06 & 0.16 & 0.46 & 0.23 & 0.56 & 0.38 & $P<0.001$ & NS \\
\hline $22: 5(g / d)$ & 0.02 & 0.02 & 0.03 & 0.05 & 0.19 & 0.08 & 0.20 & 0.12 & $P<0.001$ & NS \\
\hline $22: 6(g / d)$ & 0.11 & 0.15 & $0 \cdot 14$ & 0.29 & 0.90 & 0.40 & 1.06 & 0.69 & $P<0.001$ & NS \\
\hline PUFA/SFA & 0.37 & 0.07 & 0.39 & 0.13 & 0.50 & 0.22 & 0.50 & 0.16 & $P=0.001$ & NS \\
\hline$($ MUFA + PUFA)/SFA & $2 \cdot 04$ & 0.46 & 2.09 & 0.36 & $2 \cdot 29$ & 0.78 & $2 \cdot 12$ & 0.61 & NS & NS \\
\hline$n-6 / n-3$ PUFA & $9 \cdot 5$ & $3 \cdot 6$ & $9 \cdot 7$ & 4.9 & 4.4 & 1.7 & $5 \cdot 0$ & 3.6 & $P<0.001$ & NS \\
\hline
\end{tabular}

${ }^{*}$ For details of subjects and procedures, see the Subjects and methods section and Table 1. 
Table 4. Change (\%) in serum lipids, lipoperoxides and adhesion molecules, of the volunteers during the oily fish or red meat diets (four portions per week) ${ }^{\star}$

(Mean values and standard deviations)

\begin{tabular}{|c|c|c|c|c|c|}
\hline & \multicolumn{2}{|c|}{ Red meat diet } & \multicolumn{2}{|c|}{ Oily fish diet } & \multirow[b]{2}{*}{ ANOVA } \\
\hline & Mean & SD & Mean & SD & \\
\hline Total-c & $-2 \cdot 1$ & 1.6 & $-2 \cdot 3$ & 1.4 & NS \\
\hline LDL-c & $-5 \cdot 2$ & $2 \cdot 7$ & -7.5 & 2.9 & NS \\
\hline HDL-c & $1 \cdot 2$ & 1.4 & $7 \cdot 2$ & $2 \cdot 1$ & $P=0.023$ \\
\hline Total-c/HDL-c & $-3 \cdot 1$ & 1.6 & $-8 \cdot 0$ & $2 \cdot 3$ & NS \\
\hline LDL-c/HDL-C & $-6 \cdot 0$ & $2 \cdot 7$ & $-12 \cdot 2$ & $4 \cdot 1$ & NS \\
\hline TAG & $7 \cdot 9$ & $5 \cdot 3$ & $-3 \cdot 1$ & $4 \cdot 8$ & NS \\
\hline Lipoperoxides & $44 \cdot 1$ & $47 \cdot 1$ & -7.8 & $12 \cdot 6$ & NS \\
\hline sVCAM-1 & $8 \cdot 7$ & 4.7 & $-2 \cdot 6$ & $4 \cdot 2$ & NS \\
\hline sICAM-1 & $4 \cdot 7$ & 4.5 & $2 \cdot 8$ & 4.6 & NS \\
\hline
\end{tabular}

HDL-c, HDL-cholesterol; LDL-c, LDL-cholesterol; sICAM-1, soluble intercellular adhesion molecule-1; sVCAM-1, vascular cell adhesion molecule-1; Total-c, total cholesterol.

${ }^{*}$ For details of subjects and procedures, see the Subjects and methods section and Table 1.

no significant differences were observed in iron status parameters between the two experimental diets ${ }^{(25)}$. However, increasing oily fish consumption may induce changes in cardiovascular risk parameters and there are no data in the scientific literature on cardiovascular risk of iron-deficient women. This is the first time to our knowledge that these findings have been reported in young women with low iron stores.

The red meat diet reflects contemporary dietary habits in Spain ${ }^{(26)}$ while the oily fish diet coincides with the current Spanish guidelines ${ }^{(27)}$. PUFA intake during the oily fish diet (6.5\% of total energy intake) was between Spanish and British recommendations ${ }^{(27,28)}$, and $n-3$ PUFA intake did not exceed the 'generally recognized as safe' (GRAS) levels marked by the Food and Drug Administration ${ }^{(29)}$. In order to prevent diet-related chronic diseases, one of the population nutrient intake goals published by the WHO is for $n$-3 PUFA to represent $1-2 \%$ of the total energy intake ${ }^{(30)}$. The corresponding Spanish dietary guidelines establish that $0.5-1 \%$ of total energy should come from linolenic acid and $0 \cdot 2-0.5 \%$ from

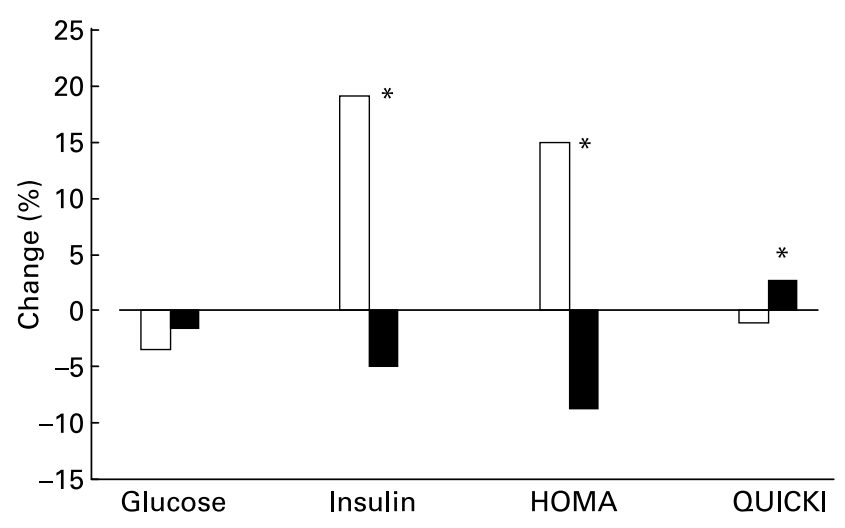

Fig. 1. Change (\%) of glucose and insulin levels, as well as Homeostasis Model Assessment (HOMA) index and Quantitative Insulin Sensitivity Check Index (QUICKI) between baseline and the end of each dietary period with oily fish ( $\square$ ) or red meat $(\square)$ diets (four portions per week). Glucose changes between dietary treatments were not significantly different. Insulin, HOMA and QUICKI changes were significantly different between dietary treatments: ${ }^{\star} P=0.030,0.050$ and 0.036 , respectively.
EPA and DHA, within a $n-6 / n-3$ ratio of $4 / 1$ to $10 / 1^{(31)}$. The present study demonstrated that it was not possible to achieve the goals for EPA and DHA intake with a diet containing five portions of red meat per week. In contrast, the oily fish diet provided tolerable upper intake levels for these two essential $n-3$ PUFA. On the other hand, both diets provided the recommended intake of $\alpha$-linolenic acid, as all participants consumed similar amounts of vegetables and legumes.

The present findings that oily fish consumption increased HDL-c and tended to reduce serum TAG coincide with those of other authors ${ }^{(10,32-34)}$. We attribute the absence of significant differences in TAG levels between dietary treatment groups to the fact that all volunteers presented the lipid profiles and TAG concentrations of healthy individuals. Data from the literature show that the most important changes observed in TAG levels after the dietary treatments were either due to a related disease of an individual volunteer ${ }^{(10,32)}$ or to $n-3$ PUFA taken in the form of dietary supplements or fish oils ${ }^{(33,34)}$.

Variations in Total-c and LDL-c have not always been observed in other studies using $n-3$ fatty acids ${ }^{(32,34)}$. Some authors even report increases in LDL-c levels after consumption of doses of $n-3$ PUFA above $1.7 \mathrm{~g} / \mathrm{d}$, most likely in subjects presenting hypertriacylglyceridaemia ${ }^{(32,33)}$ as well as an elevated susceptibility of LDL particles to oxidation $^{(32,35,36)}$. Other authors have stated that the consumption of low amounts of $n-3$ PUFA $(0.9,0.6$ or $0.3 \mathrm{~g} / \mathrm{d} n-3$ in the form of fish oil) for a long period does not significantly influence the susceptibility of LDL to oxidative modifications ${ }^{(37)}$. Supplementation with $n-3$ PUFA, as compared with n-6 PUFA or MUFA, has been reported to reduce lag time, indicating enhanced LDL oxidizability, and also the oxidation rate, suggesting decreased oxidizability of LDL particles ${ }^{(38,39)}$.

In the present study, the level of oxidation products (lipoperoxides) did not increase after an oily fish diet that provided an average of $2.75 \mathrm{~g}$ dietary $n-3$ PUFA/d, indicating that an intake of nearly $3 \mathrm{~g} \mathrm{n}-3$ PUFA from oily fish does not affect oxidation in the study participants. It is well known that excess tissue iron is associated with a high oxidative stress in iron overload conditions ${ }^{(20)}$, while these women presented low iron stores, which may have exerted a protection against oxidation in the oily fish diet.

The absence of effects of the oily fish diet on soluble adhesion molecules (sVCAM-1 and sICAM-1) is consistent with the findings of Miles et al. ${ }^{(40)}$, who observed no changes in these parameters in young and elderly individuals after fish oil consumption. Values of sVCAM-1 and sICAM-1 in the women of the present study were similar to those observed by our group in healthy post-menopausal females ${ }^{(41)}$.

As SFA intake from the two diets was similar, the high (nearly $50 \mathrm{~g} / \mathrm{d}$ ) oleic acid $(18: 1)$ intake, constituting approximately $25 \%$ of the energy consumed, of individuals on the red meat diet may partly explain the favourable results with regard to Total-c and LDL-c in this diet. Oleic acid exerts a modulating effect on lipid metabolism and reduces cardiovascular risk $^{(42)}$. Therefore, by providing extra oleic acid the red meat diet seems to have compensated for the fact that its $n-3$ PUFA content was lower than that of the oily fish diet. The fact that oleic acid is the main component of olive oil, which is a characteristic component of the Mediterranean diet and was thus consumed by all participants, may also 
have influenced the study results. Moreover, participation in the study in and of itself may have played a role in improving the dietary habits, and thus the nutritional status, of the volunteers.

As for the results in relation to insulin sensitivity, the present study confirms that a moderate consumption of oily fish beneficially affects insulin action by reducing insulin levels while maintaining pre-existing glucose concentration values, while excessive intake of fish and $n-3$ PUFA (up to $10 \mathrm{~g} / \mathrm{d}$ ) have shown deterioration of glucose control in subjects suffering from type 2 diabetes mellitus ${ }^{(43)}$.

In accordance with previous findings ${ }^{(10,43-47)}$, we attribute the positive effects of the oily fish diet to its EPA and DHA content, which is ten times higher than that of the red meat diet, while $\alpha$-linolenic acid intake was similar in both periods. It has been assessed that membrane enrichment with PUFA improves glucose uptake which is related to an increase in the levels of insulin-responsive GLUT1 and GLUT4 in the plasma membrane ${ }^{(48)}$. The ingestion of both $n-6$ and $n-3$ fatty acids has been found to suppress hepatic lipogenesis, reduce the hepatic output of TAG, enhance ketogenesis, and induce fatty acid oxidation in both the liver and the skeletal muscle ${ }^{(43,48)}$.

With regard to the effect of oleic acid on insulin sensitivity, bibliographic data indicate that it is more beneficial than SFA $^{(49)}$ although the effects of MUFA on insulin resistance have never been compared with those of PUFA ${ }^{(10,16,50)}$. The present results indicate that the oily fish diet provides $1 \cdot 5-2 \mathrm{~g}$ oleic acid/d less than the red meat diet but seems to improve insulin sensitivity by replacing it with $n-3$ fatty acids. The present results are also in agreement with a previous report indicating that middle-aged meat eaters present a lower insulin sensitivity than lacto-ovo vegetarians ${ }^{(21)}$.

To the best of our knowledge, this is the first nutritional intervention study with oily fish to report decreased insulin resistance in young iron-deficient women. This is in agreement with previous reports ${ }^{(10,43-47)}$, suggesting that regular oily fish consumption exerts a beneficial effect in men and women. However, n-3 PUFA capsules were more effective than salmon in one study carried out in overweight and obese adults who received controlled energy-restricted diets ${ }^{(47)}$, and a daily fish meal had no effect in overweight subjects unless it was incorporated into a weight-loss regimen ${ }^{(10,46)}$.

While the beneficial effects of PUFA on insulin sensitivity and glucose levels are well documented ${ }^{(14,45,50,51)}$, most of the studies carried out to date have used supplements or fish oils to include high doses of PUFA in the diet. A remarkable fact of the present study is the inclusion of a whole food as part of a healthy diet, which is an important approach in present nutrition research. Even if an isolated food component is shown to be bioactive, the whole food acts synergistically to influence the risk of chronic diseases ${ }^{(52)}$. From a public health point of view, the results obtained are relevant, giving additional support to the recommendations concerning oily fish consumption. Moreover, the present results emphasize that changes in insulin sensitivity must be shifted from the traditional glucocentric towards a lipocentric point of view ${ }^{(53)}$.

\section{Conclusion}

Changing dietary habits by increasing oily fish consumption and reducing red meat intake may protect against the development of type 2 diabetes mellitus in young women. Further studies using oily fish diets in subjects at high risk of type 2 diabetes mellitus or the metabolic syndrome should be carried out. Finally, iron-deficient women, who are advised to increase red meat intake, should be aware of the beneficial effects on insulin sensitivity of consuming oily fish within a high iron bioavailability diet.

\section{Acknowledgements}

This study was supported by the Spanish Ministry of Science and Innovation (projects AGL 2002-04411-C02-01 ALI and AGL2006-09519 ALI). S. N.-C. was the holder of a Comunidad de Madrid-European Social Fund FPI fellowship. S. S. had a research contract from the Spanish Ministry of Science and Innovation (Juan de la Cierva). S. N.-C. and A. M. P.-G. carried out the intervention study and laboratory analysis. M. P. V. was the principal investigator of the project. The article was co-written by all authors. Sardines and tuna cans were provided by Calvo SA (Spain). The authors would like to thank the volunteers, Laura Barrios for statistical support and Dr Angeles Carbajal for her dietary advice. None of the authors had any conflict of interest.

\section{References}

1. Harris WS (2007) Omega-3 fatty acids and cardiovascular disease: a case for omega-3 index as a new risk factor. Pharmacol Res 55, 217-223.

2. Panagiotakos DB, Pitsavos C, Zampelas A, et al. (2005) Fish consumption and the risk of developing acute coronary syndromes: the CARDIO2000 study. Int J Cardiol 105, 403-409.

3. Iso H, Kobayashi M, Ishihara J, et al. (2006) Intake of fish and $n-3$ fatty acids and risk of coronary heart disease among Japanese: the Japan Public Health Center-Based (JPHC) Study Cohort I. Circulation 113, 195-202.

4. Li Q, Wang M, Tan L, et al. (2005) Docosahexaenoic acid changes lipid composition and interleukin-2 receptor signaling in membrane rafts. J Lipid Res 46, 1904-1913.

5. Calder PC (2006) n-3 Polyunsaturated fatty acids, inflammation, and inflammatory diseases. Am J Clin Nutr 83, 1505S-1519S.

6. Thies F, Garry JM, Yaqoob P, et al. (2003) Association of $n-3$ polyunsaturated fatty acids with stability of atherosclerotic plaques: a randomised controlled trial. Lancet 361, 477-485.

7. Geleijnse JM, Giltay EJ, Grobbee DE, et al. (2002) Blood pressure response to fish oil supplementation: metaregression analysis of randomised trials. J Hypertens 20, 1493-1499.

8. Goodfellow J, Bellamy MF, Ramsey MW, et al. (2000) Dietary supplementation with marine omega-3 fatty acids improves systemic large artery endothelial function in subjects with hypercholesterolemia. J Am Coll Cardiol 35, 265-270.

9. Harris WS (1997) n-3 Fatty acids and serum lipoproteins: human studies. Am J Clin Nutr 65, 1645-1654.

10. Mori TA, Bao DQ, Burke V, et al. (1999) Dietary fish as a major component of a weight-loss diet: effect on serum lipids, glucose, and insulin metabolism in overweight hypertensive subjects. Am J Clin Nutr 70, 817-825.

11. Harris WS \& Bulchandani D (2006) Why do omega-3 fatty acids lower serum triacylglycerols? Curr Opin Lipidol 17, 387-393.

12. Wallace TM \& Matthews DR (2002) The assessment of insulin resistance in man. Diabet Med 19, 527-534. 
13. Colditz GA, Manson JE, Stampfer MJ, et al. (1992) Diet and risk of clinical diabetes in women. Am J Clin Nutr 55, 1018-1023.

14. Feskens EJM, Loeber JG \& Kromhout D (1994) Diet and physical activity as determinants of hyperinsulinemia: The Zutphen Elderly Study. Am J Epidemiol 140, 350-360.

15. Borkman M, Chisholm DJ, Furler SM, et al. (1989) Effects of fish oil supplementation on glucose and lipid metabolism in NIDDM. Diabetes 38, 1314-1319.

16. Vessby B, Uusitupa M, Hermansen K, et al. (2001) Substituting dietary saturated for monounsaturated fat impairs insulin sensitivity in healthy men and women: the KANWU study. Diabetologia 44, 312-319.

17. Delarue J, LeFoll C, Corporeau C, et al. (2004) n-3 Long chain polyunsaturated fatty acids: a nutritional tool to prevent insulin resistance associated to type 2 diabetes and obesity? Reprod Nutr Dev 44, 289-299.

18. Delarue J, Couet C, Cohen R, et al. (1996) Effects of fish oil on metabolic responses to oral fructose and glucose loads in healthy humans. Am J Physiol 270, 353-362.

19. Stene LC, Ulriksen J, Magnus P, et al. (2000) Use of cod liver oil during pregnancy associated with lower risk of type I diabetes in the offspring. Diabetologia 43, 1093-1098.

20. Facchini F \& Saylor KL (2002) Effect of iron depletion on cardiovascular risk factors. Studies in carbohydrate-intolerant patients. Ann N Y Acad Sci 967, 342-351.

21. Hua NW, Stoohs RA \& Facchini F (2001) Low iron status and enhanced insulin sensitivity in lacto-ovo vegetarians. Br J Nutr 86, $515-519$.

22. Moher D, Schultz KF \& Altman DG (2001) The CONSORT statement: revised recommendations for improving the quality of reports of parallel-group randomised trials. Lancet 357, 1191-1194.

23. Friedewald WT, Levy RI \& Fredrickson DS (1972) Estimation of the concentration of low-density lipoprotein cholesterol in plasma, without use of the preparative ultracentrifuge. Clin Chem 18, 499-502.

24. Navas-Carretero S, Pérez-Granados AM, Sarriá B, et al. (2008) Oily fish increases iron bioavailability of a phytate rich meal in young iron deficient women. J Am Coll Nutr 27, 96-101.

25. Navas-Carretero S, Sarriá B, Pérez-Granados AM, et al. (2006) Use of a fish versus a red meat-based diet in the prevention of iron deficiency anemia. A pilot study in young women. In Bioavailability 2006. Optimizing Dietary Strategies for Better Health in Developing Countries, p. 148 [Y Kalambaheti and S Charoenkiatkul, editors]. Chiang Mai: Mahidol University.

26. Instituto Nacional de Estadística (INE) (2007) Encuestas de presupuestos familiares. http://www.ine.es/inebmenu/indice.htm (accessed May 2007).

27. Aranceta J \& Serra-Majem L (2001) Estructura general de las guías alimentarias para la población española. Decálogo para una dieta saludable. In Guías alimentarias para la población española, pp. 183-194. Madrid: Sociedad Española de Nutrición Comunitaria, IM\&C.

28. British Nutrition Foundation (1992) Unsaturated Fatty Acids: Nutritional and Physiological Significance: The Report of the British Nutrition Foundation's Task Force. London: Chapman \& Hall.

29. Department of Health and Human Service, US Food and Drug Administration (2007) Substances affirmed as generally recognized as safe: menhaden oil. Federal Register, June 5, 1997. 62, 108: 30751-757. http://frwebgate.access.gpo.gov/cgi-bin/ getdoc.cgi?dbname $=1997$ _register\&docid $=$ fr05jn97-5 $($ accessed May 2007).

30. FAO/WHO (1998) Fats and Oils in Human Nutrition. Report of a Joint FAO/WHO Expert Consultation. FAO Food and Nutrition Paper no. 57. Rome: Food and Agriculture Organization of the United Nations.
31. Ros E (2001) Guía para una alimentación cardiosaludable. Aporte de grasa. In Guías alimentarias para la población española, pp. 413-421. Madrid: Sociedad Española de Nutrición Comunitaria, IM\&C.

32. Finnegan YE, Minihane AM, Leigh-Firbank EC, et al. (2003) Plant- and marine-derived $n-3$ polyunsaturated fatty acids have differential effects on fasting and postprandial blood lipid concentrations and on the susceptibility of LDL to oxidative modification in moderately hyperlipidemic subjects. Am J Clin Nutr 77, 783-795.

33. Kris-Etherton PM, Harris WS \& Appel LJ (2002) Fish consumption, fish oil, omega-3 fatty acids, and cardiovascular disease. Circulation 106, 2747-2757.

34. Rice R (1988) Fish oils - their significance to human health. $J$ R Soc Med 81, 499-501.

35. Pedersen H, Petersen M, Major-Pedersen A, et al. (2003) Influence of fish oil supplementation on in vivo and in vitro oxidation resistance of low-density lipoprotein in type 2 diabetes. Eur J Clin Nutr 57, 713-720.

36. Lapointe A, Couillard C \& Lemieux S (2006) Effects of dietary factors on oxidation of low-density lipoprotein particles. $J$ Nutr Biochem 17, 645-658.

37. Higgins S, Carroll YL, McCarthy SN, et al. (2001) Susceptibility of LDL to oxidative modification in healthy volunteers supplemented with low doses of $n-3$ polyunsaturated fatty acids. Br J Nutr 85, 23-31.

38. Turini ME, Crozier GL, Donnet-Hughes A, et al. (2001) Shortterm fish oil supplementation improved innate inmunity, but increased ex vivo oxidation of LDL in man - a pilot study. Eur J Nutr 40, 56-65.

39. Lee YS \& Wander RC (2005) Reduced effect on apoptosis of 4-hydroxyhexenal and oxidized LDL enriched with $n-3$ fatty acids from postmenopausal women. J Nutr Biochem 16, 213-221.

40. Miles EA, Thies F, Wallace FA, et al. (2001) Influence of age and dietary fish oil on plasma soluble adhesion molecule concentrations. Clin Sci 100, 91-100.

41. Schoppen S, Pérez-Granados AM, Carbajal A, et al. (2004) A sodium-rich bicarbonated mineral water reduces cardiovascular risk in postmenopausal women. J Nutr 134, 1058-1063.

42. Covas MI (2007) Olive oil and the cardiovascular system. Pharmacol Res 55, 175-186.

43. Martín de Santa Olalla L, Sánchez-Muniz FJ \& Vaquero MP (2009) $n$-3 Fatty acids in glucose metabolism and insulin sensitivity. Nutr Hosp 24, (In the Press).

44. Browning LM, Krebs JK, Moore CS, et al. (2007) The impact of long chain $n-3$ polyunsaturated fatty acid supplementation on inflammation, insulin sensitivity and CVD risk in a group of overweight women with an inflammatory phenotype. Diabetes Obes Metab 9, 70-80.

45. Griffin MD, Sanders T, Davies IG, et al. (2006) Effects of altering the ratio of dietary $n-6$ to $n-3$ fatty acids on insulin sensitivity, lipoprotein size, and postprandial lipemia in men and postmenopausal women aged 45-70 y: the OPTILIP Study. Am J Clin Nutr 84, 1290-1298.

46. Abete I, Parra D, Crujeiras AB, et al. (2008) Specific insulin sensitivity and leptin responses to a nutritional treatment of obesity via a combination of energy restriction and fatty fish intake. J Hum Nutr Diet 21, 591-600.

47. Ramel A, Martinez A, Kiely M, et al. (2008) Beneficial effects of long-chain $n-3$ fatty acids included in an energy-restricted diet on insulin resistance in overweight and obese European young adults. Diabetologia 51, 1261-1268.

48. Nugent C, Prins JB, Whitehead JP, et al. (2001) Arachidonic acid stimulates glucose uptake in 3T3-L1 adipocytes by increasing GLUT1 and GLUT4 levels at the plasma membrane. Evidence for involvement of lipoxygenase metabolites and 
peroxisome proliferator-activated receptor gamma. J Biol Chem 276, 9149-9157.

49. Paniagua JA, Gallego de la Sacristana A, Sánchez E, et al. (2007) A MUFA-rich diet improves posprandial glucose, lipid and GLP-1 responses in insulin-resistant subjects. J Am Coll Nutr 26, 434-444.

50. Vessby B, Tengblad S \& Lithell H (1994) Insulin sensitivity is related to the fatty acid composition of serum lipids and skeletal muscle phospholipids in 70-year-old men. Diabetologia 37, 1044-1050.
51. Storlien LH, Jenkins AB, Chisholm DJ, et al. (1991) Influence of dietary fat composition on development of insulin resistance in rats. Relationships to muscle triglyceride and omega-3 fatty acids in muscle phospholipid. Diabetes 40, 280-289.

52. Jacobs DR \& Steffen LM (2003) Nutrients, foods, and dietary patterns as exposures in research: a framework for food synergy. Am J Clin Nutr 78, 508S-513S.

53. Mittra S, Bansal VS \& Bhatnagar PK (2008) From a glucocentric to a lipocentric approach towards metabolic syndrome. Drug Discov Today 13, 211-218. 